Report No.: ORNL/TM-2000/295

\title{
THE EFFECT OF NEUTRON AND GAMMA RAY CROSS TALK BETWEEN PLASTIC SCINTILLATION DETECTORS
}

S. A. Pozzi

R. B. Oberer

L. G. Chiang

October 2000

Prepared by the

Oak Ridge National Laboratory

Oak Ridge, Tennessee 37831

managed by

UT-Battelle, LLC

for

U.S. Department of Energy

under contract DE-AC05-00OR22725 


\section{DISCLAIMER}

This report was prepared as an account of work sponsored by an agency of the United States government. Neither the United States Government nor any agency thereof, nor any of their employees, makes any warranty, express or implied, or assumes any legal liability or responsibility for the accuracy, completeness, or usefulness of any information, apparatus, product, or process disclosed, or represents that its use would not infringe privately owned rights. Reference herein to any specific commercial product, process, or service by trade name, trademark, manufacturer, or otherwise, does not necessarily constitute or imply its endorsement, recommendation, or favoring by the United States Government or any agency thereof. The views and opinions of authors expressed herein do not necessarily state or reflect those of the United States Government or any agency thereof. 


\author{
Sara A. Pozzi* \\ Richard B. Oberer \\ Lisa G. Chiang \\ Oak Ridge National Laboratory \\ P. O. Box 2008 \\ Oak Ridge, TN 37831-6004 USA \\ *Department of Nuclear Engineering \\ Polytechnic of Milan \\ Italy
}

\author{
Prepared by \\ OAK RIDGE NATIONAL LABORATORY \\ Oak Ridge, Tennessee 37831-6004 \\ managed by \\ UT-BATTELLE, LLC \\ for the \\ U. S. DEPARTMENT OF ENERGY \\ under contract DE-AC05-00OR22725
}


This page intentionally left blank. 


\section{CONTENTS}

Page

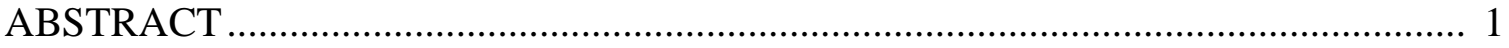

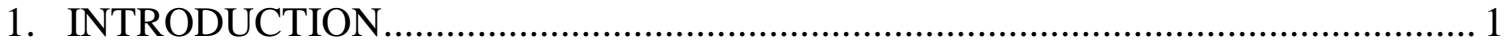

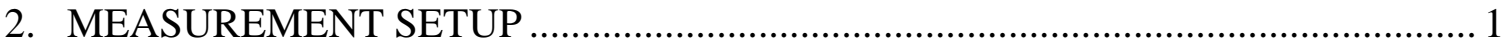

3. MEASUREMENT RESULTS AND ANALYSIS .................................................. 2

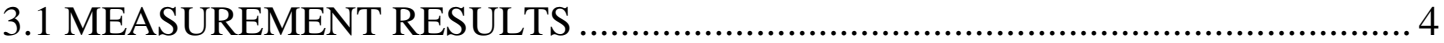

3.2 FEATURES FROM THE BICOVARIANCE .................................................. 6

3.3 MORE ON THE ${ }^{252}$ CF FISSION - NEUTRON - NEUTRON TRIPLET ........... 7

4. EFFECTS OF NEUTRON AND GAMMA SCATTERING BETWEEN

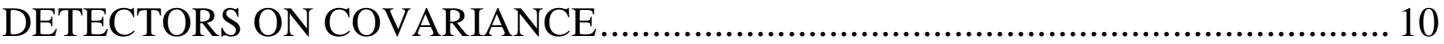

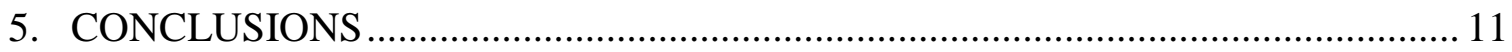

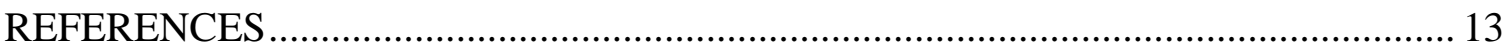


This page intentionally left blank. 


\section{LIST OF FIGURES}

Page

1 Schematic drawing of measurement setup: top view ....................................... 2

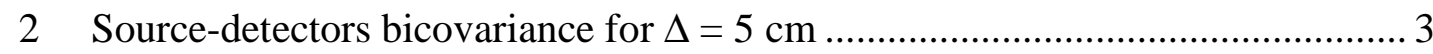

3 Source-detectors bicovariance for $\Delta=0 \mathrm{~cm}$ : top view …………..................... 4

4 Source-detectors bicovariance for $\Delta=10 \mathrm{~cm}$ : top view …………................... 5

5 Source-detectors bicovariance for $\Delta=20 \mathrm{~cm}$ : top view …………………...... 5

6 Volumes of the ridges $f-\gamma-\gamma, f-\gamma-n, f-n-n$, and $f-n-\gamma_{n}$ as a function of the distance between the detectors, $\Delta$. Lines have been drawn to guide the eye ...... 6

7 Ratio of scattered neutron energy to incident neutron energy as a function

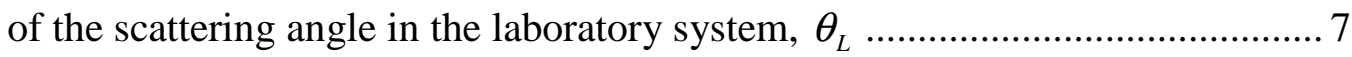

8 Source-detectors bicovariance for $\Delta=0 \mathrm{~cm}$. Boundary lines given by Eq. (2) correspond to scattering between detectors at the threshold angle, $\theta_{t h} \ldots \ldots \ldots \ldots . . . .9$

9 Source-detectors bicovariance for $\Delta=5 \mathrm{~cm}$. Boundary lines given by Eq. (2) correspond to scattering between detectors at the threshold angle, $\theta_{t h} \ldots \ldots \ldots \ldots \ldots . . .9$

10 Detector 1 - detector 2 covariance for different distances between detectors: $\Delta=0,10$, and $25 \mathrm{~cm}$ 
This page intentionally left blank. 


\begin{abstract}
In this paper a method is developed, using higher order statistics, to identify the type and degree of neutron and gamma ray cross talk between detectors that are placed in proximity to one another. A set of measurements was performed using the Nuclear Materials Identification System (NMIS) to acquire the time-dependent bicovariance of the pulses in fast plastic scintillating detectors. These signatures were analyzed to infer the degree and type of false coincidences (cross talk) in relation to true coincidences.
\end{abstract}

\title{
1. INTRODUCTION
}

In fast time-correlation measurements performed in safeguard applications ${ }^{12}$ it is common to use two or more adjacent detectors to acquire source-detector and detector-detector time-dependent correlations. These and other signatures have been $\operatorname{shown}^{7,8}$ to be sensitive to the amount of fissile mass in the sample to be analyzed. In such applications, correlated counts are due to fission events induced in the fissile material, whereby neutrons and gamma rays are emitted simultaneously and correlated in time with the neutron that induced fission. The time-correlated counts in the detectors are thus closely related to the amount of fissile material inside the sample to be analyzed. In this context, it is desirable to minimize the amount of "false" coincidences due to the scattering of neutrons and gamma rays from one detector to another. These events give timecorrelated pulses in the detectors that are not directly related to fission events, thus concealing the required signal.

Cross talk identification is of interest in many other applications in which detectors are placed close to each other and correlation measurements are performed. Such applications include the study of heavy-ion induced reactions and spectroscopy ${ }^{6,14}$ and interferometric experiments. ${ }^{2,4,13}$

In this paper higher order statistical signatures ${ }^{9}$ were collected using NMIS to infer the degree and type of neutron and gamma ray scattering between the detectors. Measurements were performed in which the distance between the source and two detectors was kept constant, and the distance between the detectors was varied.

\section{MEASUREMENT SETUP}

Active measurements were performed with NMIS in which the instrumented ${ }^{252} \mathrm{Cf}$ source was placed at a distance of one meter from the two detectors, as shown in Figure 1. The ${ }^{252} \mathrm{Cf}$ source provides a timed source of neutrons and gamma rays. Each spontaneous fission event emits, on average, four neutrons and seven to eight gamma rays. The detectors are Bicron - BC 420 fast organic scintillating crystal. The crystal dimensions are 3.75 by 3.75 by 4 inches. The sides of the detectors are shielded with $1 / 4$ inch of lead. The distance $d$ between the source and each detector was held constant at $100 \mathrm{~cm}$. Six measurements were performed, with the distance between detectors $\Delta$ equal to 0,5 , $10,15,20$, and $25 \mathrm{~cm}$. By increasing the distance between the detectors the solid angle between them decreases. Therefore more cross talk should occur for smaller $\Delta$ and less 
for larger $\Delta$. Conversely, true coincidences from the multiple particles emitted by ${ }^{252} \mathrm{Cf}$ are independent of the distance between the detectors because the solid angle subtended by the detectors to the source does not change. There is however some angular dependence between the fission neutrons. ${ }^{15}$ For example, the angle between fission neutrons is more likely to be $180^{\circ}$ than $90^{\circ}$. This effect is not significant in this experiment because the distance $d$ between the source and detector is large compared to the distance $\Delta$ between detectors. Therefore the angle between true coincident neutrons is always small.

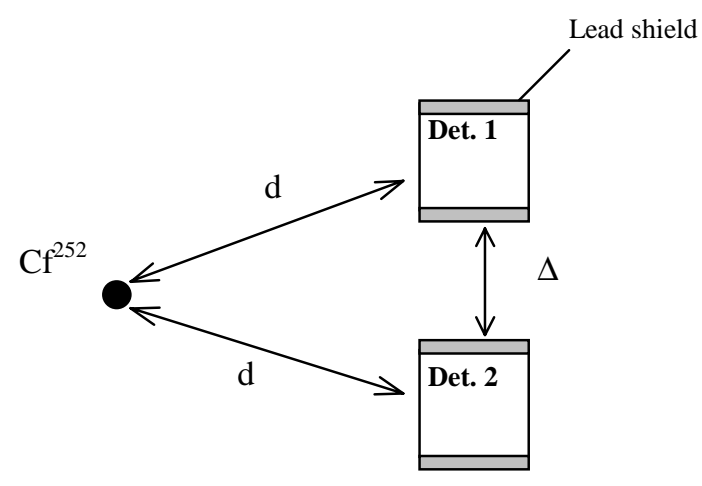

Fig. 1. Schematic drawing of measurement setup: top view.

\section{MEASUREMENT RESULTS AND ANALYSIS}

The source-detector-detector bicovariance consists of pairs of counts in the two detectors correlated with a ${ }^{252} \mathrm{Cf}$ fission. This signature is shown in Figure 2 for $\Delta=5 \mathrm{~cm}$. The time at which a pulse occurs in detector 1 after a source fission is $\tau_{1}$, and $\tau_{2}$ is the time at which it occurs in detector 2 after a source fission.

The following are the possible triplets of events that contribute to the bicovariance:

1. $f-\gamma-n$ and $f-n-\gamma$

2a. f-n-n' and f-n'-n

2b. $\mathrm{f}-\mathrm{n}_{1}-\mathrm{n}_{2}$

3. $\mathrm{f}-\mathrm{n}-\gamma_{\mathrm{n}}$ and $\mathrm{f}-\gamma_{\mathrm{n}}-\mathrm{n}$

4. $\mathrm{f}-\boldsymbol{\gamma}-\boldsymbol{\gamma}$

where $\mathrm{f}$ is a ${ }^{252} \mathrm{Cf}$ fission, $\gamma$ is a fission gamma detection, $\gamma_{\mathrm{n}}$ is a (n, $\gamma$ ) gamma detection, and $\mathrm{n}$ is a neutron detection. The order of the triplets represents a ${ }^{252} \mathrm{Cf}$ fission, detection in detector 1 and detection in detector 2 respectively. The pair $\mathrm{n}_{1}-\mathrm{n}_{2}$ indicates two different neutrons, while the pair n-n' indicates the same neutron before and after scattering.

The features in Figures 2 and 3 are numbered according to the possible triplets of events given above. In feature 1, a gamma pulse in one detector is followed by a neutron pulse 
from the same fission in the second detector. For example, a gamma ray reaches detector 1 at $\tau_{1}$ equal to $3.3 \mathrm{~ns}$, while a neutron reaches detector 2 at $\tau_{2}$ greater than $\tau_{1}$, varying according to the spectrum of the neutrons emitted by ${ }^{252} \mathrm{Cf}$. This occurrence gives the ridge along axis $\tau_{2}$. The opposite is also possible, giving a second, symmetric ridge along axis $\tau_{1}$.

In features $2 \mathrm{a}$ and $2 \mathrm{~b}$, two neutron pulses in the two detectors form the triplet. This instance can be given by two different neutrons, $\mathrm{n}_{1}-\mathrm{n}_{2}$, generated by the same ${ }^{252} \mathrm{Cf}$ fission event as in $2 b$ or by the scattering of one neutron from one detector to the other, $\mathrm{n}$-n', as in 2a. These two different cases give rise to two components: the first gives a small, broad distribution oriented in the center of the $\tau_{1}, \tau_{2}$ plane $(2 b)$, while the second gives two distinct, symmetric ridges (2a).

A neutron generating a gamma ray in one detector $n-\gamma_{n}$ that gives a pulse in the other detector produces feature 3 . The secondary gamma rays can be generated by a $(\mathrm{n}, \mathrm{n}$ ' $\gamma)$ reaction or a $(\mathrm{n}, \gamma)$ reaction. The result is a ridge along the $\tau_{1=} \tau_{2}$ line because the gamma ray reaches the second detector almost instantaneously.

Finally, fission-gamma-gamma triplets $\mathrm{f}-\gamma-\gamma$, labeled 4 , can be originated by two distinct gamma rays or by a single gamma ray scattering from one detector to the other. Either process results in similar $\tau_{1}-\tau_{2}$ pairs. These two processes therefore cannot be resolved as in the $n-n$ cases between feature $2 a$ and $2 b$ of the bicovariance.

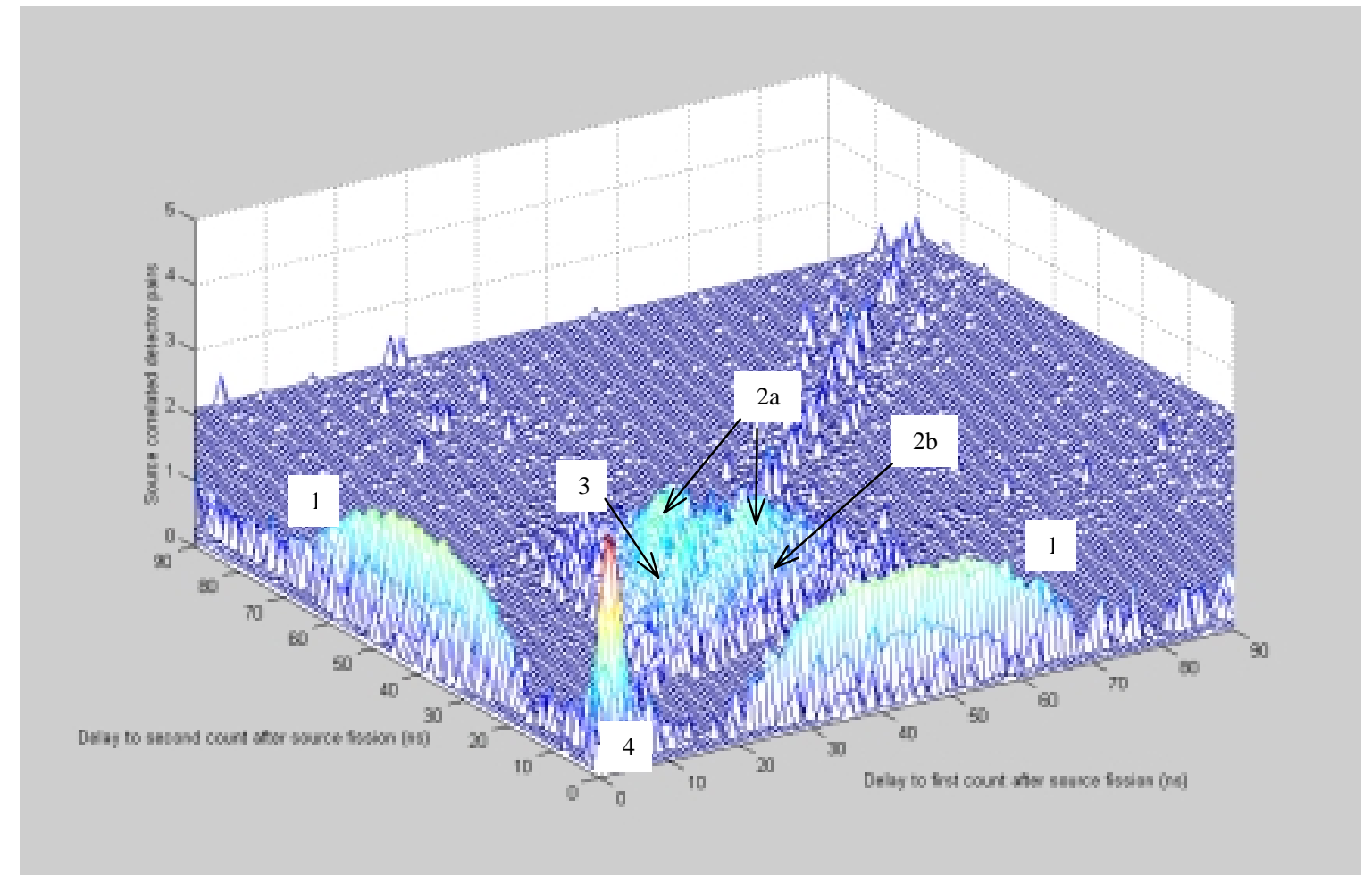

Fig. 2. Source-detectors bicovariance for $\Delta=5 \mathrm{~cm}$. 


\subsection{MEASUREMENT RESULTS}

Figures 3 through 5 show the top view of the source-detector bicovariances for $\Delta=0,10$, and $20 \mathrm{~cm}$ respectively. Inspection of these figures shows that some of the ridges depend on the detector-detector distance $\Delta$ whereas others do not. The fission-gamma neutron ridges, $\mathrm{f}-\boldsymbol{\gamma}-\mathrm{n}$, (labeled 1) are not affected by changes in $\Delta$. This is because there are no $(\gamma, \mathrm{n})$ processes present. The fission-neutron-neutron ridges, f-n-n' (labeled 2a) move and decrease as $\Delta$ increases. On the other hand, the $\mathrm{f}-\mathrm{n}_{1}-\mathrm{n}_{2}$ feature is only mildly dependent on $\Delta$. The $f-\gamma_{\mathrm{n}} \mathrm{n}$ ridge (labeled 3 ) and the fission gamma-gamma ridges, $\mathrm{f}-\boldsymbol{\gamma}-\gamma($ labeled 4 ) do not change position although their volume changes, as will be shown in the Section 3.2 .

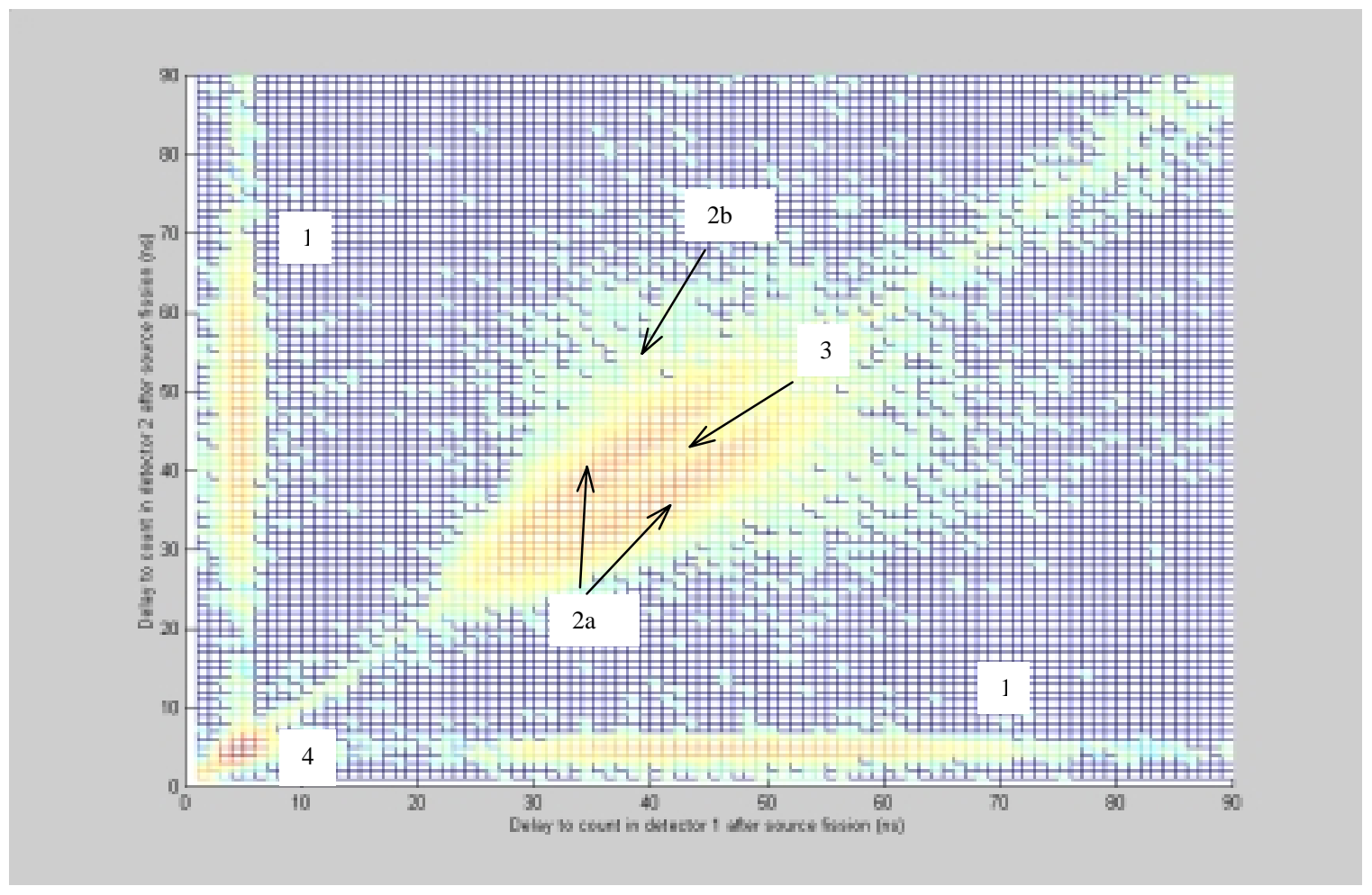

Fig. 3. Source-detectors bicovariance for $\Delta=0 \mathrm{~cm}$ : top view. 


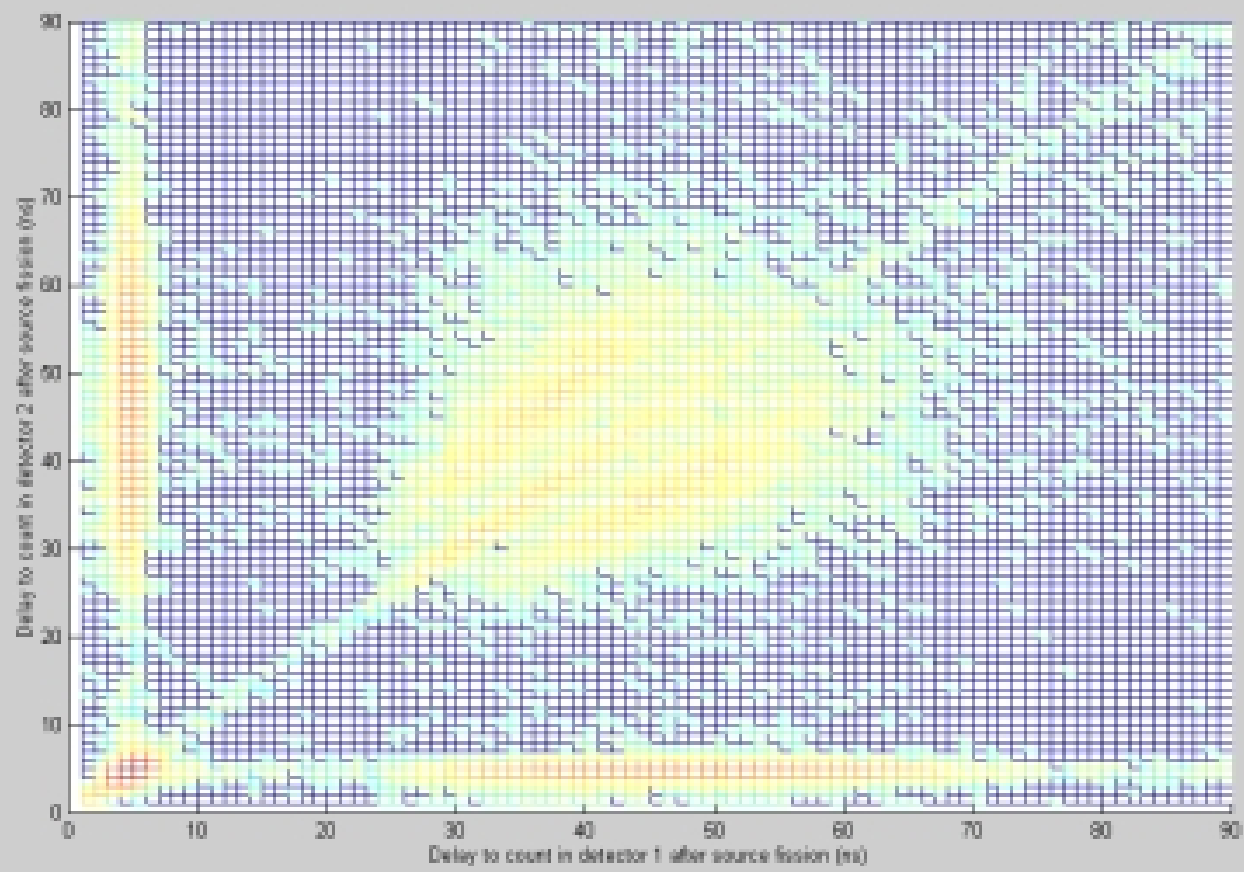

Fig. 4. Source-detectors bicovariance for $\Delta=10 \mathrm{~cm}$ : top view.

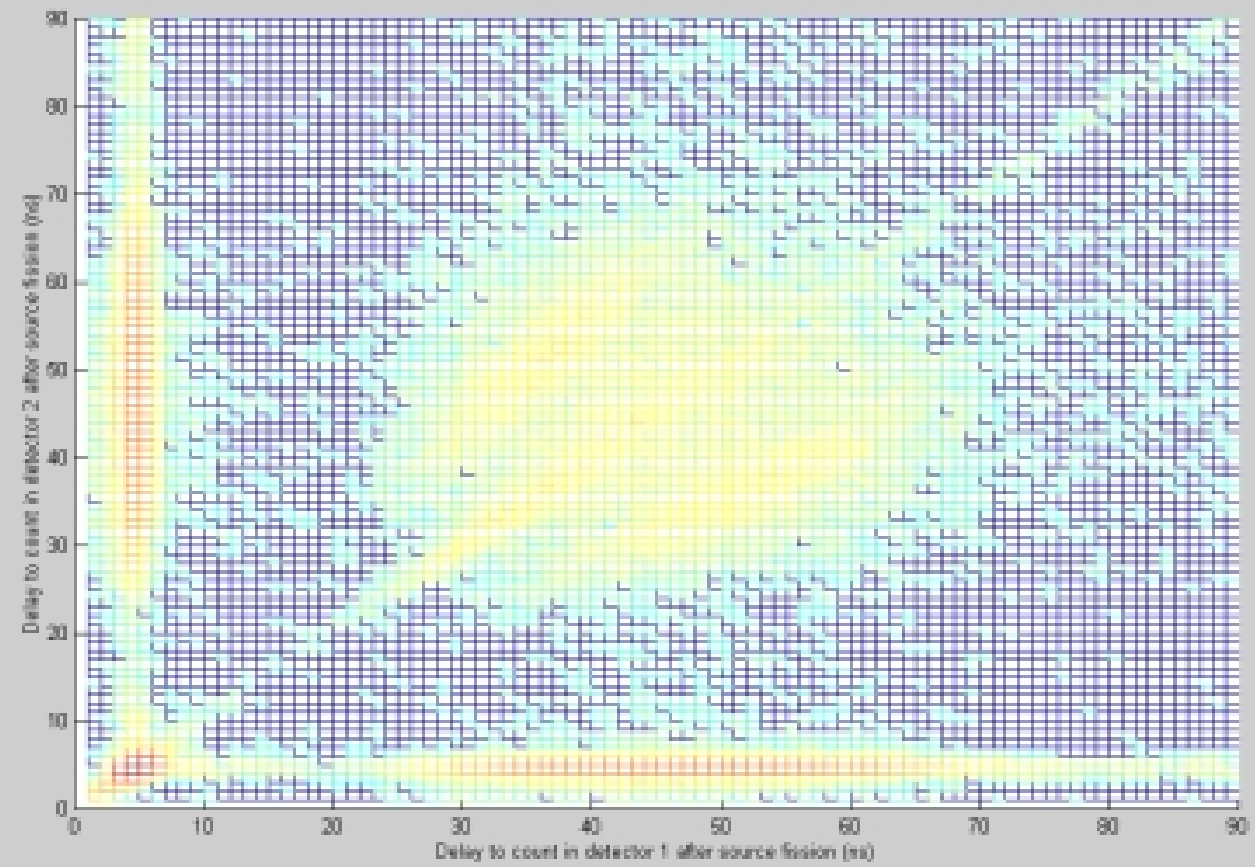

Fig. 5. Source-detectors bicovariance for $\Delta=20 \mathrm{~cm}$ : top view. 


\subsection{FEATURES FROM THE BICOVARIANCE}

Figure 6 shows the volumes of the ridges given by the $f-\gamma-\gamma, f-\gamma-n, f-n-n$, and $f-n-\gamma_{n}$ triplets as a function of the distance between the detectors, $\Delta$. As can be seen, only $\mathrm{f}-\boldsymbol{\gamma}-\mathrm{n}$ triplets are independent of the distance between detectors, indicating that scattering does not affect this particular signature.

On the other hand $f-\gamma-\gamma, f-\gamma-n$, f-n-n', and $f-n-\gamma_{n}$ triplets are strongly affected by scattering. The volume of the ridge given by these triplets increases approximately by a factor of two for the $f-\gamma-\gamma$ triplet and a factor fourteen for the $f-n-n$ ', and $f-n-\gamma_{n}$ triplets.

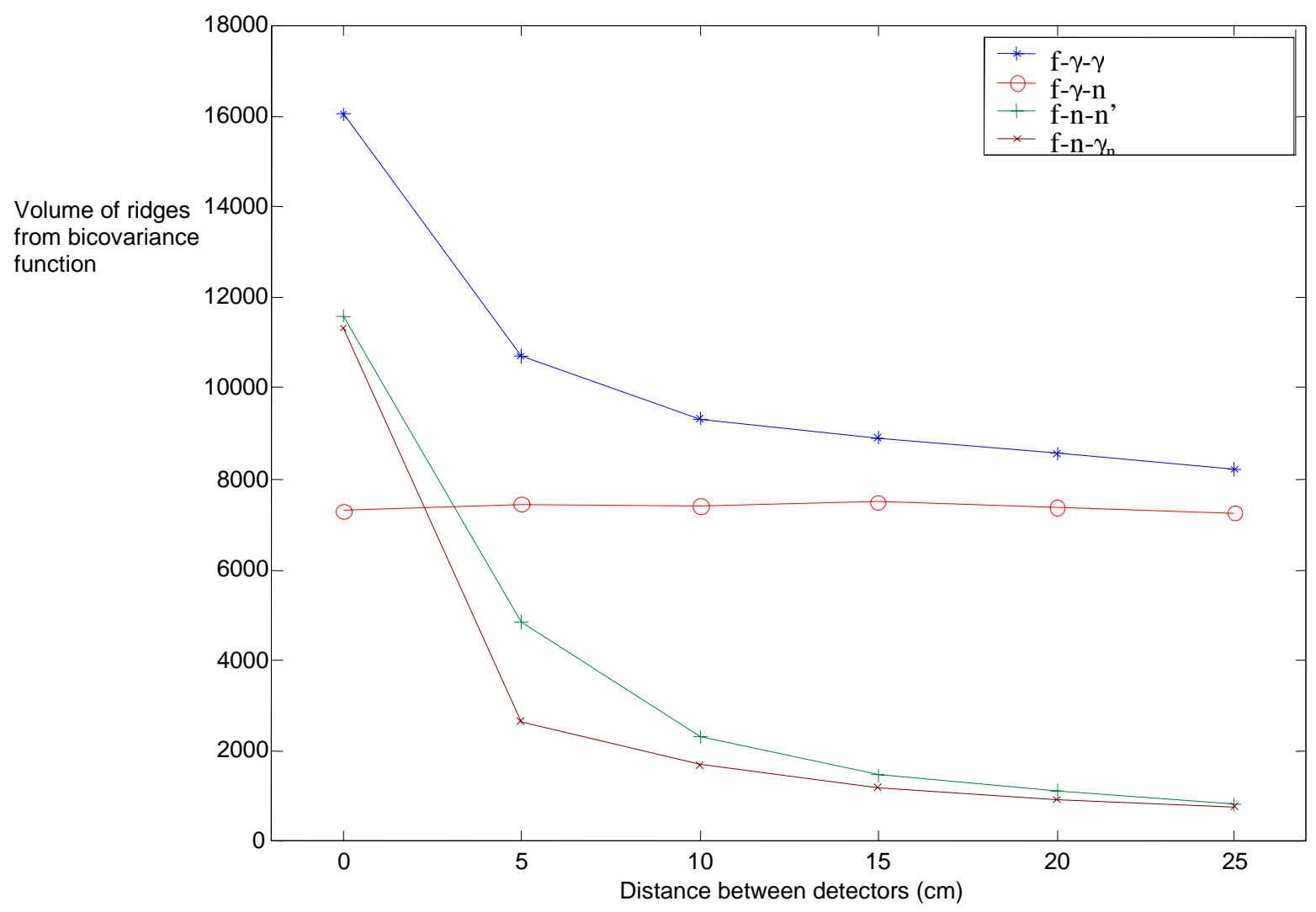

Fig. 6. Volumes of the ridges $f-\gamma-\gamma, f-\gamma-n, f-n-n$, and $f-n-\gamma_{n}$ as a function of the distance between the detectors, $\Delta$. Lines have been drawn to guide the eye. 


\subsection{ANALYSIS OF THE ${ }^{252}$ CF f-n-n' TRIPLET}

The f-n-n' scattering process can be modeled based on a single hydrogen scattering assumption. From this model the outer boundary of the f-n-n' ridges can be predicted. Let $\tau_{1}$ and $\tau_{2}$ be the times after a ${ }^{252} \mathrm{Cf}$ source fission at which a detection occurs in detector 1 and detector 2, respectively. In the discussion, the following assumptions are made:

1. scattering occurs on $\mathrm{H}$ only, ${ }^{3}$ and

2. only one scattering event occurs before the neutron escapes the detectors.

Let $E_{1}$ be the neutron energy before detection in detector 1. The energy after the first collision is

$$
E_{2}=E_{1} \cos ^{2} \theta_{L}
$$

where $\theta_{L}$ is the scattering angle in the laboratory system. A plot of the ratio of the energy of the scattered neutron to that of the incident neutron is given in Figure 7. Neutron scattering on hydrogen is forwardly directed in the lab system.

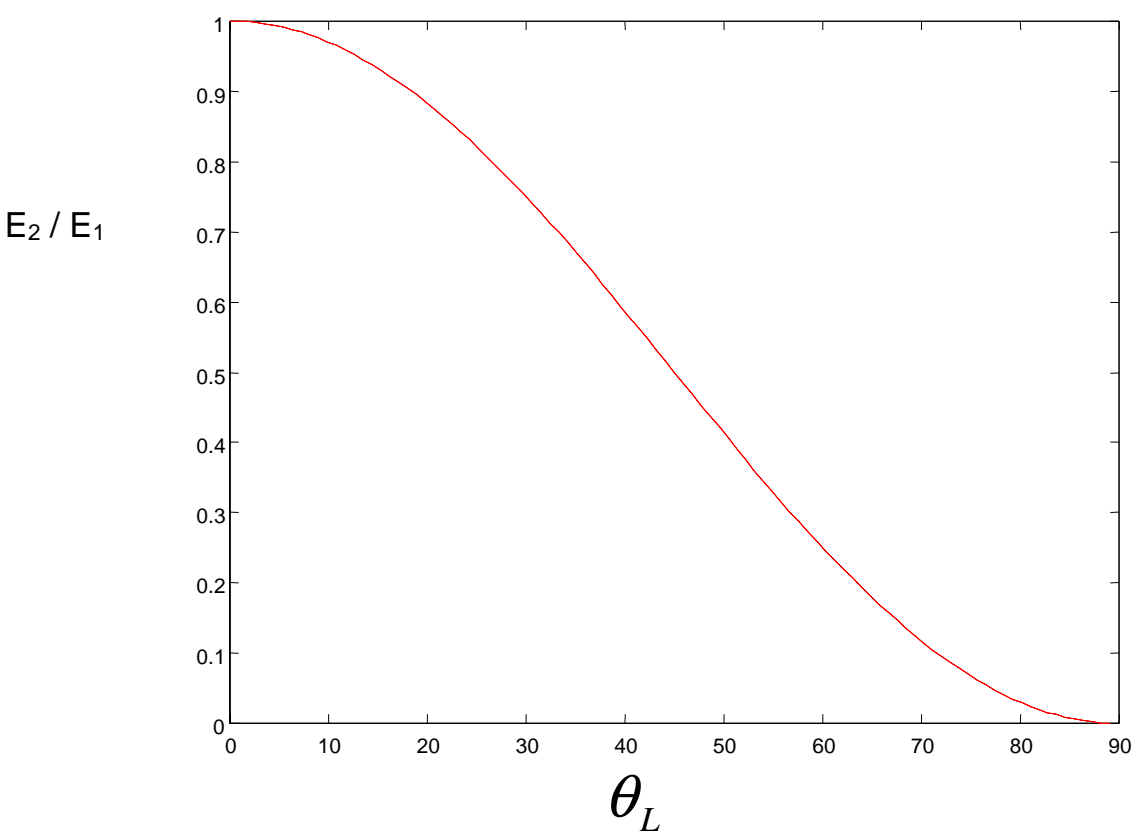

Fig. 7. Ratio of scattered neutron energy to incident neutron energy as a function of the scattering angle in the laboratory system, $\theta_{L}$. 
The relationship between energies allows us to write a relationship between $\tau_{1}$ and $\tau_{2}$

$$
\tau_{2}=\tau_{1}\left(1+\frac{d_{2}}{d_{1}} \frac{1}{\cos \theta_{L}}\right)
$$

where $d_{1}$ and $d_{2}$ are the distances from the source to the first collision and from the first to the second collision respectively.

For a count to be recorded in the detectors, the neutron must deposit an energy exceeding the threshold value set in the constant fraction discriminator (CFD). This minimum energy corresponds to a maximum scattering angle $\theta_{\text {th }}$ given by

$$
\theta_{t h}=\cos ^{-1} \sqrt{E_{t h} / E_{1}} .
$$

A neutron scattered at an angle greater than $\theta_{t h}$ cannot register a pulse in the second detector because it will have insufficient energy. In our experiment, the CFD discriminator level was set at $40 \mathrm{mV}$, corresponding ${ }^{1}$ to a neutron energy threshold of $1.16 \mathrm{MeV}$. Using Eq. (3) with $\mathrm{E}_{1}=2 \mathrm{MeV}$, for example, gives $\theta_{t h}=40.4^{\circ}$. For larger values of $\Delta$, neutrons scattering once in the first detector at angles less than $\theta_{t h}$ cannot hit the second detector.

Substituting $\theta_{t h}$ into Eq. (2) allows one to determine the relationship between detection times that is shown with solid black lines in Figures 8 and 9. These lines are the outer boundary of the fission-neutron-neutron cross talk ridges, and correspond to the longest delay between counts in the two detectors for a single hydrogen scatter. 


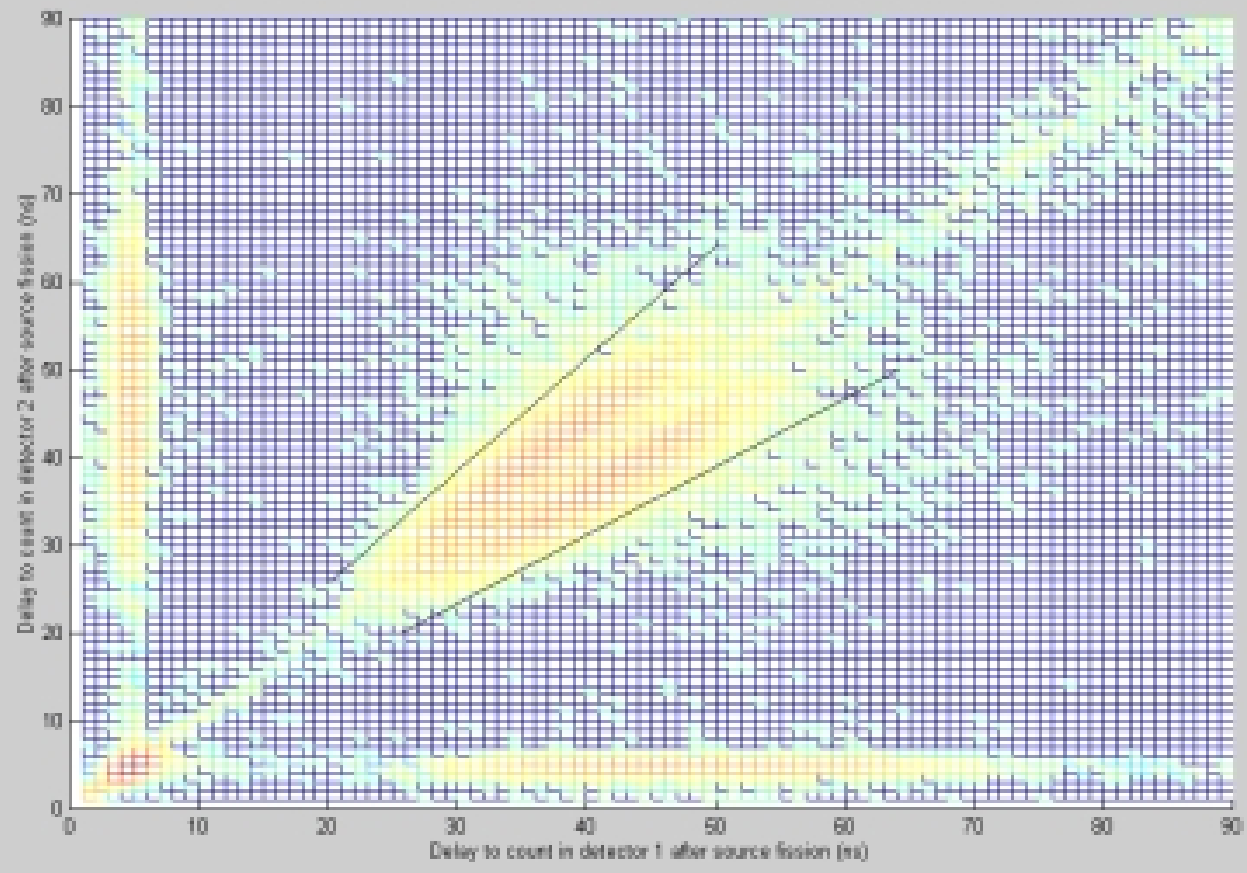

Fig. 8. Source-detectors bicovariance for $\Delta=0 \mathrm{~cm}$. Boundary lines given by Eq. (2) correspond to scattering between detectors at the threshold angle, $\theta_{t h}$.

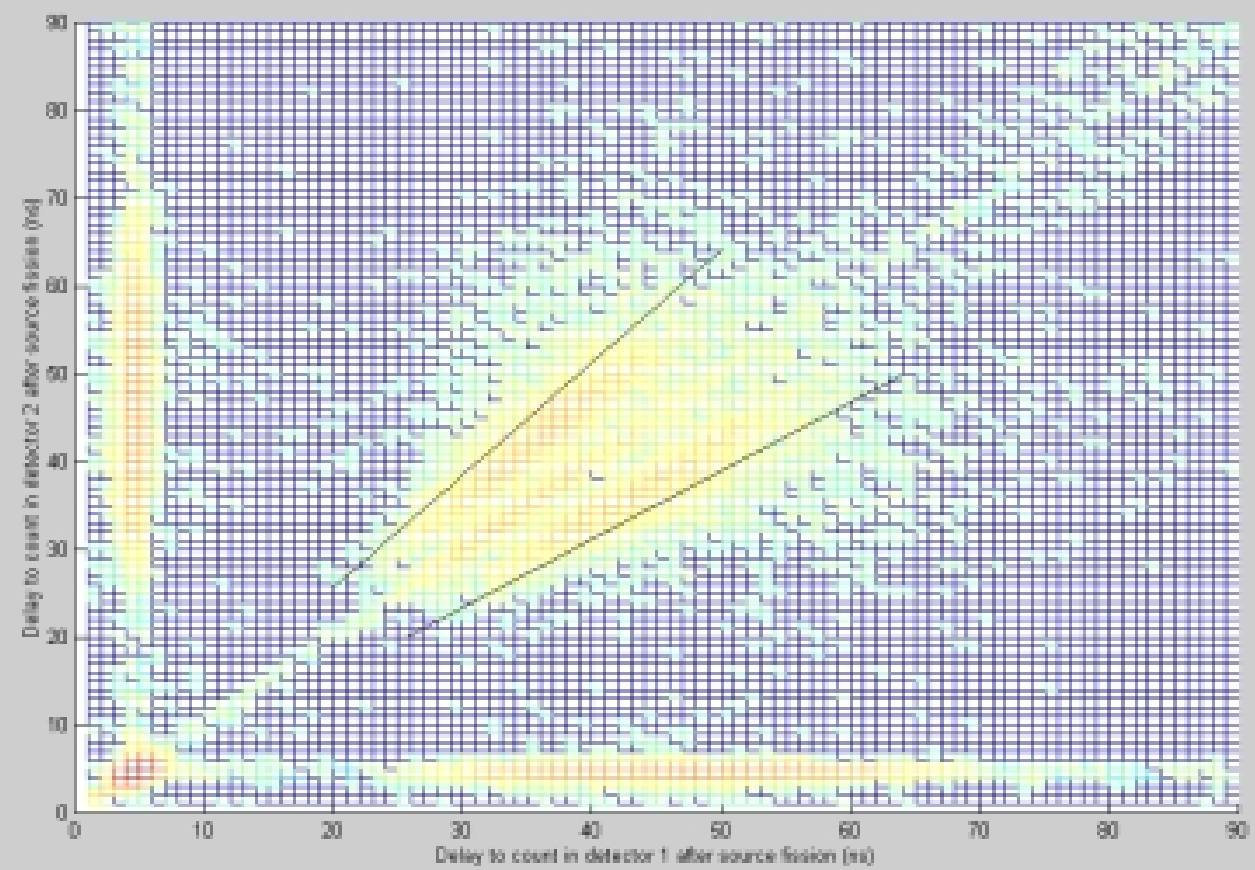

Fig. 9. Source-detectors bicovariance for $\Delta=5 \mathrm{~cm}$. Boundary lines given by Eq. (2) correspond to scattering between detectors at the threshold angle, $\theta_{t h}$. 


\section{EFFECTS OF NEUTRON AND GAMMA SCATTERING BETWEEN DETECTORS ON COVARIANCE}

Source-detector and the detector-detector covariances are among the signatures acquired by NMIS. These signatures have been shown to be sensitive to sample enrichment. The effect of cross-talk on these signatures can be divided into two categories. The first is due to particles that are unrelated to the source fission (for example gamma rays coming from the deexcitation of the nuclei of the sample or background radiation). The second is due to particles that are time-correlated to the source. The use of higher order statistics ${ }^{11}$ to correlate detector pulses to the initiating source fission can eliminate the effect of the first type of scattering, but not the effect of the second.

Detector-detector covariances were acquired with NMIS for varying distances between detectors $\Delta$, in the measurement configuration described in Section 2. Figure 10 shows the detector-detector covariances for different values of $\Delta$. The combined effect of $\gamma-\gamma$ and $n-\gamma_{n}$ scattering between detectors is evident in the gamma peak. The $n-n$ ' scattering gives two symmetric peaks. In typical operation, $\Delta=0 \mathrm{~cm}$ and this effect appears as shoulders to the gamma peak. As expected, $\gamma$-n and $n-\gamma$ pairs are not affected by scattering.

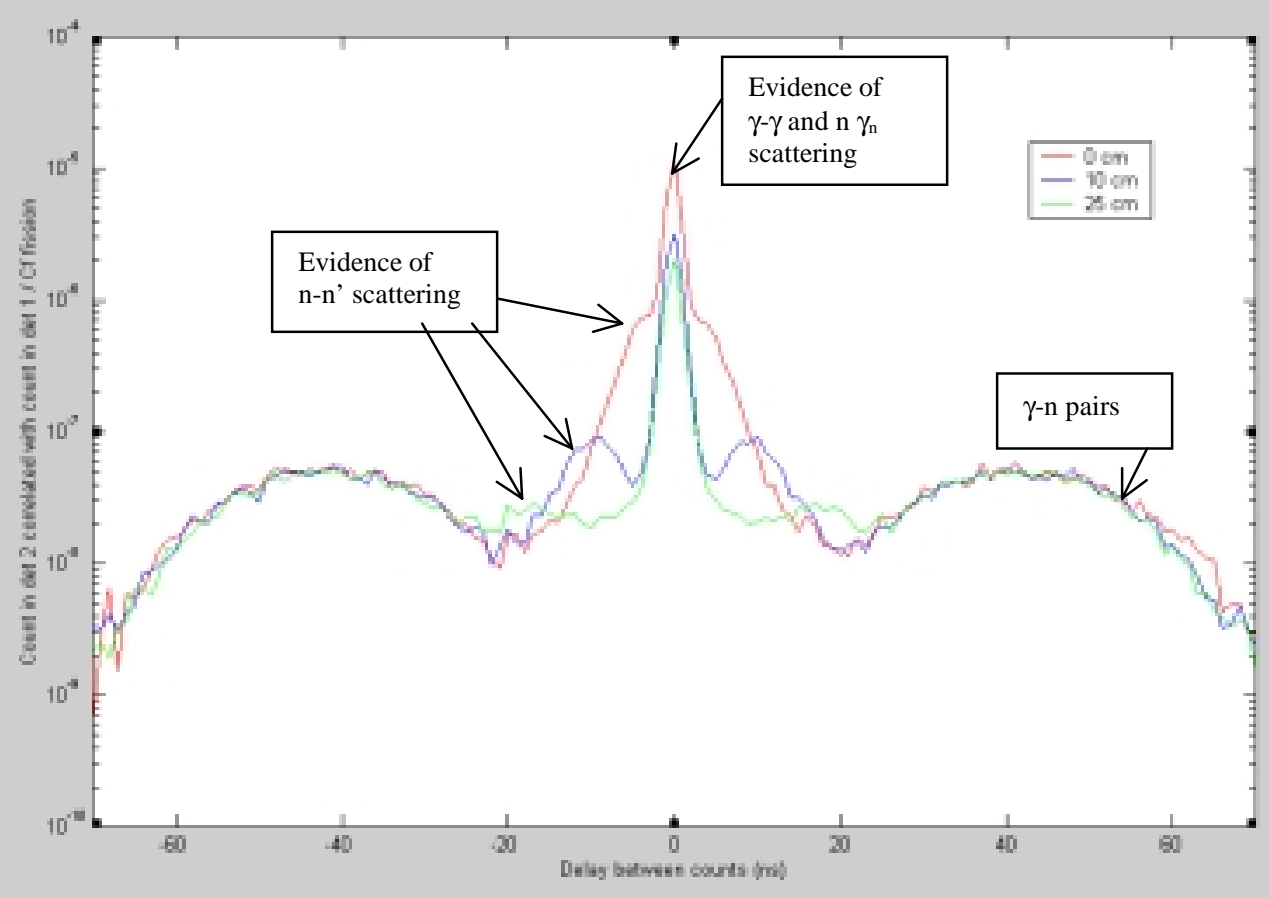

Fig. 10. Detector 1 - detector 2 covariance for different distances between detectors: $\Delta=0,10$, and $25 \mathrm{~cm}$. 


\section{CONCLUSIONS}

In this paper measurements performed with NMIS have been used to quantify the interaction between fast plastic scintillating detectors used in time-correlation measurements. By maintaining a constant distance between source and detectors, and varying the distance between detectors we showed that both neutron and gamma ray cross talk contribute significantly to the observed signatures. Furthermore, gamma rays that are generated by neutron interactions in one detector can proceed to give correlated pulses in the second detector.

These effects are considerable and must be taken into account in determining the rate of true coincidences in measurements that make use of detectors placed close to each other. 
This page intentionally left blank. 


\section{REFERENCES}

1. L. G. Chiang, R. B. Oberer, and S. A. Pozzi, Method to Correlate CFD Discriminator Level and Energy Deposition by Neutrons and Photons in a Fast Plastic Scintillating Detector, ORNL/TM-2000/193, Oak Ridge National Laboratory (2000).

2. P. Desesquelles, A. J. Cole, A. Dauchy, A. Giorni, D. Heuer, A. Lleres, C. Morand, J. Saint-Martin, P. Stassi, J. B. Viano, B. Chambon, B. Cheynis, D. Drain, and C. Pastor, Cross Talk and Diaphony in Neutron Detectors, Nuclear Instruments and Methods in Physics Research A307 (1991) 366.

3. M. Drosg, D. M. Drake, and P. Lisowski, The Contribution of Carbon Interactions to the Neutron Counting Efficiency of Organic Scintillators, Nuclear Instruments and Methods, 176 (1980).

4. R. Ghetti, N. Colonna, and J. Helgesson, Influence of Cross-Talk Rejection Procedures on Two-Neutron Intensity Interferometry, Nuclear Instruments and Methods in Physics Research A421 (1999) 542.

5. H. Gotoh and H. Yagi, Solid Angle Subtended by a Rectangular Slit, Nuclear Instruments and Methods 96 (1971) 485.

6. A. V. Kuznetsov, I. D. Alkhazov, D. N. Vakhtin, V. G. Lyapin, V. A. Rubchenya, W. H. Trzaska, K. Loberg, and A. V. Daniel, Neutron Multi-Detector System: Mutual Influence of its Modules, Nuclear Instruments and Methods in Physics Research A346 (1994) 259.

7. J. K. Mattingly T. E. Valentine, and J. T. Mihalczo, NWIS Measurements For Uranium Metal Annular Castings, Y/LB-15,971, Oak Ridge Y-12 Plant (1998).

8. J. K. Mattingly, Enhanced Identification of Fissile Assemblies Via of Application of High Order Statistical Signatures, Y/LB-15,978, Oak Ridge Y-12 Plant (1998).

9. J. K. Mattingly, High Order Statistical Signatures from Source-Driven Measurements of Subcritical Fissile Systems, Y/LB-15,966/R1, Oak Ridge Y-12 Plant (1998).

10. J. K. Mattingly, J. A. March-Leuba, J. T. Mihalczo, R. B. Perez, L. G. Chiang, T. E. Valentine, and J. A. Mullens, Passive NMIS Measurements to Estimate the Shape of Plutonium Assemblies, Y/LB-16,012, Oak Ridge Y-12 Plant (1999). 
11. J. K. Mattingly, J. T. Mihalczo, T. E. Valentine, and R. B. Perez, Reduction of Background by Higher Order Correlations with NMIS, Institute for Nuclear Materials Management, $41^{\text {st }}$ Annual Meeting, July 16-20, 2000, New Orleans, Louisiana.

12. J. T. Mihalczo, T. E. Valentine, and J. K. Mattingly, NWIS Methodology, Y/LB15,953, Oak Ridge Y-12 Plant (1997).

13. J. Pluta, G. Bizard, P. Desesquelles, A. Dlugosz, O. Dorvaux, P. Duda, D. Durand, B. Erazmus, F. Hanappe, B. Jakobsson, C. Lebrun, F. R. Lecolley, R. Lednicky, P. Leszczynski, K. Mikhailov, K. Miller, B. Noren, T. Pawlak, M. Przewlocki, O. Skeppstedt, A. Stavinsky, L. Stuttge, B. Tamain, and K. Wosinska, Two-Neutron Interferometry Measurements, Nuclear Instruments and Methods in Physics Research A411 (1998) 417.

14. J. Wang, A. Galonsky, J. J. Kruse, P. D. Zecher, F. Deak, A. Horvath, A. Kiss, Z. Seres, K. Ieki, and Y. Iwata, Neutron Cross-Talk in a Multi-Detector System, Nuclear Instruments and Methods in Physics Research A397 (1997) 380.

15. R. Vandenbosch and J. R. Huizenga, Nuclear Fission, Academic Press, N.Y. (1973). 